\title{
JOINT MULTICHANNEL PANSHARPENING FOR MULTISPECTRAL IMAGERY
}

\author{
Hussein A. Aly ${ }^{*, \dagger}$, Gaurav Sharma* \\ *ECE Dept., University of Rochester, Rochester, NY 14627-0126 \\ ${ }^{\dagger}$ Military Technical college (MTC), Ministry of Defense, Cairo, Egypt
}

\begin{abstract}
Pan-sharpening is a common post-processing operation for captured multispectral satellite imagery, where the spatial resolution of images gathered in various spectral bands is enhanced by fusing them with a panchromatic image captured at a higher resolution. Previously proposed pan-sharpening techniques operate on a per-channel basis, sharpening each multispectral band independently based on the panchromatic image, often in an ad hoc manner. In contrast with most prior techniques, we formulate pan-sharpening as the problem of jointly estimating the high resolution multispectral images to minimize the combined squared residual error in physically motivated observation models of the low resolution multispectral and the high resolution panchromatic images. To realize pan-sharpening using our proposed formulation, we develop an iterative algorithm to solve the joint minimization resulting in an overall algorithm with modest computational complexity. We evaluate our proposed algorithm and benchmark it against previously proposed methods using established quantitative measures of SNR, SAM, ERGAS, Q, and Q4 indices. Both the quantitative results and visual evaluation demonstrate that the proposed joint formulation provides superior results compared with pre-existing methods.
\end{abstract}

Index Terms - pan-sharpening, satellite imagery, image fusion, spectral imaging

\section{INTRODUCTION}

For reasons of cost, bandwidth, and to maintain adequate image quality in the presence of noise, satellite based multi and hyper spectral image capture systems use on-board imaging sensors that vary in spatial resolution: typical sensor configurations, capture a high resolution panchromatic image spanning a wide spectral band and lower resolution images for individual spectral bands. For applications using the satellite imagery, once data is received on the ground, the spectral band images are post-processed to obtain versions that match the higher resolution sampling of the panchromatic image. This process, commonly referred to as pan-sharpening, merges together low resolution and spectral information captured in spectral channels with high resolution detail from the panchromatic image.

Several image pan-sharpening methods have been proposed in the literature. A majority of these techniques operate using a component or subband substitution framework where the multispectral and the panchromatic images are mapped into a transform domain and in the transform domain, some component or subband data of the panchromatic image is inserted or used to replace the data in the multispectral image, and the inverse transform is then applied to this modified transform domain spectral image to obtain the pan-sharpened version. Common examples in the substitution framework include: a) methods based purely on "color", i.e. channel transforms, such as intensity-hue-saturation (IHS) substitution [1-3], Brovey transform, principal component replacement, Gram-Schmidt transform [4], and b) methods based on spatial transforms, such as the multi-scale wavelet decomposition replacement [5,6], multiwavelet transform [7], and the curvelet transform [8]. The substitution approach is largely ad hoc, although some variants, such as the Gram-Schmidt transform, are motivated by improved spectral consistency and the parameter choices in the spatial transform based approaches can be motivated by the relative spatial bandwidths of the capture bands. Besides the substitution framework, pan-sharpening has also been addressed in a modelbased method that is spectrally consistent [9] and using a more structured restoration approach to the problem [10]. Additional variants that do not fit into one of the pre-existing categories include, compressive image fusion for pan-sharpening [11], dictionary learning based pan-sharpening [12] and smoothing filter-based intensity modulation [13]. Studies comparing the different pan-sharpening methods have also previously been presented in [14-18].

In this paper, we propose a new formulation for the pansharpening problem. We pose the problem of estimating the high resolution multispectral images, jointly, as the minimization of a combined squared residual error in physically motivated observation models of the low resolution multispectral and the high resolution panchromatic images. We then develop an iterative algorithm to solve the minimization with modest computational complexity. Compared with prior methods for pan-sharpening, the proposed approach presents novelty in the combination of three respects: a) the pan-sharpening of the multispectral channels is performed jointly, rather than independently for each channel, b) upsampling is explicitly incorporated in the formulation instead of utilizing interpolated versions of the multispectral images to model the multispectral observation, and c) the objective function that we minimize simultaneously imposes spectral and spatial consistency with the observed data. Results benchmarking the method and comparing the proposed method against the leading existing alternatives demonstrate its advantage: it offers superior performance in both visual comparison and in numerical metrics used for assessment of quality.

The rest of this manuscript is organized as follows. Section 2 introduces our joint formulation of the pan-sharpening problem as a minimization problem. Section 3 develops an iterative minimization approach for solving the minimization in a computationally tractable fashion. Experimental results obtained using the proposed algorithm are presented in Section 4. Concluding remarks and a discussion bring the paper to a close in Section 5.

\section{JOINT FORMULATION OF PAN-SHARPENING}

We begin with a physical model for the multispectral imaging system. A spatio-spectral distribution $r[\chi, v ; \lambda]$ of light intensity is incident upon the sensor image planes used for capturing the panchromatic and the multispectral images ${ }^{1}$, where the pair $(\chi, v)$ represents

\footnotetext{
${ }^{1}$ Throughout this paper, we assume that the captured images are spatially registered using suitable techniques.
} 
an orthogonal coordinate system for the sensor image plane aligned with the sensor sampling grid and $\lambda$ denotes the wavelength of light. A panchromatic image is obtained, using a sensor sensitive to a wide wavelength range ${ }^{2}$ and having a sampling interval $X$ along each dimension, represented as a 2-D orthogonal lattice $\Lambda$. The captured panchromatic image is represented as $p[\mathbf{x}], \mathbf{x} \in \Lambda$, where

$$
p[\chi, v]=\mathcal{H}^{p}[\chi, v] * \int r[\chi, v ; \lambda] \tau^{p}(\lambda) d \lambda,
$$

with $\mathcal{H}^{p}[\chi, v]$ and $\tau^{p}(\lambda)$ representing, respectively, the point spread function (PSF) and the spectral responsivity of the panchromatic imager, and $*$ representing the convolution operation. Simultaneously, $K$ spectral images are also acquired via imagers sensitive to narrow $^{3}$ spectral bands on a sparser orthogonal sampling lattice $\Gamma$ having a spatial-sampling interval $q X$ along each dimension, where $q>1$ so that the spectral channels have a lower resolution than the panchromatic. These captured spectral images are represented by $c_{i}[\mathbf{x}], \mathbf{x} \in \Gamma$, for $i=1,2, \ldots K$, where

$$
c_{i}[\chi, v]=\mathcal{H}_{i}[\chi, v] * \int r[\chi, v ; \lambda] \tau_{i}(\lambda) d \lambda,
$$

with $\mathcal{H}_{i}[\chi, v]$ and $\tau_{i}(\lambda)$ representing, respectively, the point spread function (PSF) and the spectral responsivity of the $i^{\text {th }}$ spectral imager. When the panchromatic image $p$ and the spectral images $\left\{c_{i}\right\}_{i=1}^{K}$ are acquired by the same satellite, $q$ is typically an integer factor and $\Gamma \subset \Lambda$, which is the situation we focus on in this paper.

Given the observed high resolution panchromatic image $p[\mathbf{x}], \mathbf{x} \in$ $\Lambda$ and the low resolution spectral images $\left\{c_{i}[\mathbf{x}]\right\}_{i=1}^{K}, \mathbf{x} \in \Gamma$, our objective is to recover high resolution spectral images $\left\{f_{i}[\mathbf{x}]\right\}_{i=1}^{K} \mathbf{x} \in$ $\Lambda$, where

$$
f_{i}[\chi, v]=\mathcal{H}_{i}[\chi / q, v / q] * \int r[\chi, v ; \lambda] \tau_{i}(\lambda) d \lambda .
$$

Here, we assume that the PSF for the desired high resolution image is defined by scaling the PSF for the actually captured image by a factor $(1 / q)$ along each spatial direction, so that the corresponding modulation transfer function, and effective bandwidth, are scaled by a factor $q$, as is desirable when the sampling density is increased by $q$ (along each dimension).

The problem of estimating the high resolution spectral images $\left\{f_{i}[\mathbf{x}]\right\}_{1=1}^{K}, \mathbf{x} \in \Lambda$, is a special version of the upsampling problem [19], where, unlike typical single image super-resolution, the panchromatic image $p[\mathbf{x}], \mathbf{x} \in \Lambda$ can provide information missing in the captured spectral imagery making conventional regularization unnecessary. To proceed to formulate the pan-sharpening problem, we specify the observation model for the low-resolution observed spectral images in the discrete domain as a low-pass filter $h_{i}[\mathbf{x}]$ on the lattice $\Lambda$ followed by subsampling on the lattice $\Gamma$, which is illustrated in Fig. 1. Using the standard stacked notation [20, pp. 212], we obtain the operation in matrix-vector format as

$$
\mathbf{c}_{i}=\mathbf{H}_{i} \mathbf{f}_{i}+\boldsymbol{\eta}_{i}
$$

where $\mathbf{c}_{i}$ and $\mathbf{f}_{i}$ are the stacked notation vectors representing $c_{i}[\mathbf{x}]$ and $f_{i}[\mathbf{x}]$, respectively, and $\mathbf{H}_{i}$ is the rectangular matrix representing the low-pass filtering and sub-sampling, having essentially one row for every $q^{2}$ columns ${ }^{4}$, and $\eta_{i}$ represents the noise in stacked format. If the filter $\mathcal{H}_{i}[\chi, v]$ is an ideal band-limited filter matched

\footnotetext{
${ }^{2}$ The Landsat7 panchromatic imager, for example, covers the wavelength range from 0.52 to 0.92 micrometers.

${ }^{3}$ Relative, to the panchromatic channel.

${ }^{4}$ Because our final implementations are all based on discrete filtering operations, we leave unspecified the sizes of the images and therefore also the corresponding vectors and matrices.
}

to the Nyquist bandwidth for the lattice $\Gamma$, the discrete domain observation model is exact [21]. Because ideal filters are non-realizable and due to other limitations, practical systems use non-ideal filters. The filters $h_{i}[\mathbf{x}], i=1,2, \ldots K$ can then be optimally designed as in [22] using knowledge of $\mathcal{H}_{i}[\chi, v]$ provided as part of the system specifications. For our formulation, analogous to the filters $h_{i}[\mathbf{x}]$ for the multispectral channels, we also define a filter $h[\mathbf{x}]$ that models a corresponding downsampling for the panchromatic image, i.e., $h[\mathbf{x}] * p[\mathbf{x}]$ approximates capture of a low resolution panchromatic image on the lattice $\Gamma$ via a filter with impulse response $\mathcal{H}^{p}[q \chi, q v]$.

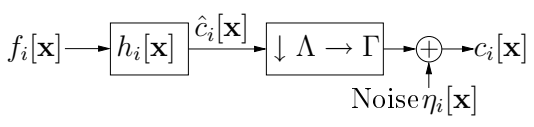

Fig. 1. Discrete domain observation model for the low resolution spectral image $c_{i}[\mathbf{x}]$ in terms of the corresponding high resolution image $f_{i}[\mathbf{x}]$.

We now formulate pan-sharpening as the estimation of $\left\{f_{i}[\mathbf{x}]\right\}_{i=1}^{K}$ for $\mathbf{x} \in \Lambda$ by combining the low resolution and partly aliased information in $\left\{c_{i}[\mathbf{x}]\right\}_{i=1}^{K}, \mathbf{x} \in \Gamma$ with the higher resolution spatial information available in the panchromatic image $p[\mathbf{x}], \mathbf{x} \in \Lambda$, exploiting, in the process, the spectral correlation between the panchromatic and the spectral channels due to their overlap. The specification of the spectral sensitivities of the panchromatic and the individual spectral channels provides a model for the spectral correlation, specifically, we write

$$
\tau^{p}(\lambda)=\sum_{i=1}^{K} w_{i} \tau_{i}(\lambda)+\zeta(\lambda),
$$

where the summation represents the best attainable approximation to the spectral responsivity $\tau^{p}(\lambda)$ for the panchromatic channel in terms of the spectral responsivities $\left\{\tau_{i}(\lambda)\right\}_{i=1}^{K}$ for the $K$ spectral channels, treated as a basis, and $\zeta(\lambda)$ represents the residual error in the approximation. The weights $\left\{w_{i}\right\}_{i=1}^{K}$ can be obtained from the specification of the spectral sensitivities via least squares regression. If the filters $\mathcal{H}^{p}[\chi, v]$ and $\mathcal{H}_{i}[\chi / q, v / q]$ are identical, one can readily see that the spectral relation in (5) induces a corresponding relation for the high resolution images

$$
p[\mathbf{x}]=\sum_{i=1}^{K} w_{i} f_{i}[\mathbf{x}]+\zeta[\mathbf{x}],
$$

where the image $\zeta[\mathbf{x}]$ is defined as the one corresponding to an imager with a virtual spectral sensitivity $\zeta(\lambda), \operatorname{PSF} \mathcal{H}^{p}[\chi, v]$, and sampling lattice $\Lambda$.

Finally, we formulate pan-sharpening as the joint optimization

$$
\begin{aligned}
& \grave{\mathbf{f}}=\arg \min _{\breve{\mathbf{f}}} \mathcal{J}(\breve{\mathbf{f}}, \mathbf{c}, \mathbf{p}), \\
& \mathcal{J} \triangleq \sum_{i=1}^{K}\left\|\mathbf{H}_{i} \mathbf{f}_{i}-\mathbf{c}_{i}\right\|^{2}+\left\|\mathbf{G}\left(\sum_{i=1}^{K} \omega_{i} \mathbf{f}_{i}-\mathbf{p}\right)\right\|^{2},
\end{aligned}
$$

where, we have use the stacked notation [20, pp. 212] to compactly represent images as the corresponding vectors and the filtering (and downsampling) operations as matrices, re-using the terms introduced in (4) and adding the notation $\mathbf{p}$ to denote the panchromatic image $p[\mathbf{x}]$ in stacked form, $\breve{\mathbf{f}}$ to jointly represent the complete set of high resolution multi-spectral images $\left\{\mathbf{f}_{i}\right\}_{i=1}^{K}$, and the matrix $\mathbf{G}$ denotes 
the complementary high pass filter for $h[\mathbf{x}]$ defined by $g[\mathbf{x}]=\delta[\mathbf{x}]-$ $h[\mathbf{x}]$, where $\delta[\cdot]$ represents the (Kronecker) delta function.

The first summation term in the objective function $\mathcal{J}$ represents the data-fidelity requirement for the $K$ observed spectral channels under the observation model of Fig 1 . The second term represents the requirement for consistency of the $K$ estimated high resolution images with the spectral correlation model for the panchromatic image in (6), where this requirement is imposed only on the images high pass filtered components. The high pass filtering is beneficial because the residual $\zeta[\mathbf{x}]$ in (6) is ignored, it is helpful not to include in the second term lower frequency components that would erroneously compete with the more accurate low frequency spectral information included through the first summation. This idea, specifically motivates the use of the complementary filter $\mathbf{G}$ for the corresponding channel in the second term in objective function in (7).

Observe that, in contrast with most prior formulations of pansharpening, the proposal in (7) poses the optimization jointly for the $K$ channels, instead of performing the pan-sharpening on a channelby-channel basis. Because this better enforces consistency across the estimated high resolution spectral images, as we shall see in the results in Section 4, it also leads to improved performance. To gain some intuition into why this is the case, note that in our proposed formulation because of the second term, high frequency spatial detail in the panchromatic image $p[\mathbf{x}]$ must be appropriately apportioned to the spectral channels and cannot be excessively re-utilized nor unduly ignored. Also, unlike most prior approaches, potential aliasing in the process of mapping $f_{i}[\mathbf{x}], \mathbf{x} \in \Lambda$ to $c_{i}[\mathbf{x}], \mathbf{x} \in \Gamma$ is comprehended via the observation model. This allows for the aliasing to be partly resolved via the high frequency information contributed by the panchromatic image instead of being treated as noise in the data fidelity term. Also note that the objective function combines the dual goals of spectral and spatial consistency with the observed data.

\section{ITERATIVE MINIMIZATION ALGORITHM}

The optimization problem in (7) is challenging because of the large number of variables ${ }^{5}$ corresponding to the number of spectral channels times the number of pixels in each high resolution spectral image. To address this challenge, we develop a gradient-descent [23] based iterative algorithm for optimization along with an efficient filter based implementation. Specifically, via relatively straightforward algebra, we obtain the gradient of our objective function from which, it follows that the gradient descent iteration with a numerical step size $\Delta T$ can be written in per-channel (although still coupled) form as

$$
\begin{aligned}
\mathbf{f}_{i}^{(n+1)}= & \mathbf{f}_{i}^{(n)}-\Delta T \\
& \left(\mathbf{H}_{i}^{T}\left(\mathbf{H}_{i} \mathbf{f}_{i}^{(n)}-\mathbf{c}_{i}\right)+\omega_{i} \mathbf{G}^{T} \mathbf{G}\left(\sum_{j=1}^{K} \omega_{j} \mathbf{f}_{j}^{(n)}-\mathbf{p}\right)\right),
\end{aligned}
$$

where $\cdot{ }^{T}$ denotes matrix transpose, and the superscript $\cdot{ }^{(n)}$ denotes the iteration index. The algorithm is initialized by setting $f_{i}^{(0)}$ to the image obtained by interpolation of $c_{i}$ for $i=1,2 \ldots N$, specifically bicubic interpolation in our implementation. The iterations are implemented as filtering and down/up sampling operations resulting in an overall efficient implementation of the gradient descent iterations. Space constraints prevent us from presenting the block diagram representation of the implementation and we limit ourselves

\footnotetext{
${ }^{5}$ For imagery from the IKONOS satellite imaging system, a typical set of four spectral images, represented at the panchromatic resolution at typically captured image sizes, has over 400 million pixels in aggregate.
}

to the ensuing verbal sketch of the implementation. The filtering equivalents corresponding to $\mathbf{G}$ and the filtering and downsampling interpretation for $\mathbf{H}_{i}$ have already been discussed. The operation $\mathbf{G}^{T}$ is a convolution matrix corresponding to the space-reversed PSF $g[-\mathbf{x}]$; if $g$ is quad-symmetric $(g[\mathbf{x}]=g[-\mathbf{x}])$, as imposed by the design constraint in [22], then $\mathbf{G}^{T}=\mathbf{G}$. The matrix $\mathbf{H}_{i}^{T}$ represents up-sampling from $\Gamma$ to $\Lambda$ followed by convolution with the space reversal filter $h_{i}[-\mathbf{x}]$.

\section{RESULTS}

To evaluate our proposed pan-sharpening method, we use imagery from the IKONOS multispectral imaging satellite [24], for which details of the system specifications, including the spectral responsivities and point-spread function characteristics are publicly available [25] along with a limited number of image datasets comprised of registered pan and multispectral images at their native capture resolutions. Together these data provide an ideal test set for evaluating our proposed algorithm and for benchmarking its performance against previously proposed pan-sharpening alternatives. The IKONOS satellite has five imaging channels: a panchromatic channel $p[\mathbf{x}]$ with a nominal ground resolution of $X=1 \mathrm{~m}$ along each dimension and spanning the spectral range ${ }^{6}$ from 525.8 through $928.5 \mathrm{~nm}$ and $K=4$ multispectral bands with a nominal ground resolution of $4 X=4 m$ along each dimension (i.e., $q=4$ ) and having the following spectral bandwidths: (a) MS-1 (Blue) $c_{1}[\mathbf{x}]$, $444.7-516.0 \mathrm{~nm}$, (b) MS-2 (Green) $c_{2}[\mathbf{x}], 506.4-595.0 \mathrm{~nm},(\mathrm{c})$ MS-3 (Red) $c_{3}[\mathbf{x}], 631.9-697.7 \mathrm{~nm}$, and (d) MS-4 (VNIR) $c_{4}[\mathbf{x}]$, $757.3-852.7 \mathrm{~nm}$. For processing and computation, the 11 bit pixel data was converted to a floating point values by a linear mapping with the digital value of 2047 represented as 1.0. Output images were linearly mapped to an 8 bit 0 to 255 scale to facilitate viewing and comparison on common 8 bit display systems. For computing numerical benchmarks for comparing different methods, required "ground truth" was generated in the standard manner [10,26] by lowpass filtering and downsampling the panchromatic and the spectral data by a factor of 4 along dimension using the system MTF parameters, where the lower resolution versions are then used as the observations and the higher resolution panchromatic data serves as the ground truth.

Required parameters for our pan-sharpening algorithm are obtained as follows. Using the publicly available specification data for the spectral sensitivities with least squares regression, we obtain the weights $^{7} w_{1}=0.040, w_{2}=0.175, w_{3}=0.214$ and $w_{4}=0.339$, for our iterative gradient descent algorithm the step size was empirically determined to $\Delta T=4$ and to conservatively assure convergence we used 100 iterations, after verifying that the improvement in the objective function leveled off around 75 iterations.

We compare the performance of the proposed algorithm with bicubic interpolation and two of the leading methods for pansharpening: generalized IHS [3] and multiscale wavelet model [6], where two versions of the latter are utilized with 3 and 4 levels, respectively, for the wavelet decomposition. The methods are compared using signal to noise power ratio (SNR) on a per channel basis and also several of the multi-channel measures commonly used in the remote sensing community. These latter measures are often motivated by the need to maintain relative differences in the spectral bands, which when viewing channels three at a time correspond to color hues, and therefore also evaluate the spectral consistency

\footnotetext{
${ }^{6}$ Stated spectral bandwidths correspond to full-width at half-max.

${ }^{7}$ Although these weights are obtained purely from the spectral responsivity data for the sensors, they are close in values to those obtained in [27] by regression over a set of sample images.
} 


\begin{tabular}{|c|c|c|c|c|c|c|c|c|}
\hline Pan-sharpening & SAM & ERGAS & \multicolumn{4}{|c|}{ SNR (dB) } & $Q_{32}^{\mathrm{avg}}$ & $Q_{32}^{4}$ \\
\hline Algorithm & (deg) & & $\mathrm{R}$ & $\mathrm{G}$ & B & NIR & & \\
\hline Bicubic Interpolation & 3.6 & 2.86 & 19.12 & 20.72 & 23.93 & 13.86 & 0.554 & 0.519 \\
\hline GIHS method & 6.5 & 4.82 & 15.91 & 17.37 & 15.81 & 11.28 & 0.643 & 0.754 \\
\hline $\begin{array}{l}\text { Multiscale Wavelet: } \\
\text { model } M 23 \text { levels [6] }\end{array}$ & 3.9 & 2.98 & 17.89 & 18.92 & 18.56 & 16.06 & 0.602 & 0.760 \\
\hline $\begin{array}{l}\text { Multiscale Wavelet: } \\
\text { model } M 24 \text { levels [6] }\end{array}$ & 4.4 & 3.33 & 16.78 & 17.63 & 16.88 & 15.97 & 0.571 & 0.772 \\
\hline Proposed method & 3.3 & 2.64 & 16.89 & 20.60 & 25.50 & 17.31 & 0.738 & 0.829 \\
\hline
\end{tabular}

Table 1. Quantitative performance measures of different pan-sharpening methods for the IKONOS sample data images "China-Sichuan 58205_0000000.20001003" obtained from GeoEye [24]. The metrics are computed over the irregular shaped support for valid data in the image file consisting of $2,618,026$ pixels.

of the pan-sharpened images $\grave{\mathbf{f}}_{i}$ to the acquired spectral images $\mathbf{c}_{i}$ jointly across the different spectral bands. Specifically, the metrics we use for our benchmarking are as follows. The spectral angle mapper (SAM) defined as

$$
\operatorname{SAM}(\grave{f}, \hat{c}) \triangleq \frac{1}{N} \sum_{m, n} \arccos \left(\frac{<\grave{\mathbf{f}}(m, n), \mathbf{f}(m, n)>}{\|\grave{\mathbf{f}}(m, n)\|_{2}\|\mathbf{f}(m, n)\|_{2}}\right),
$$

where $<,>$ denotes inner product of spectral components and $N$ the number of pixels in each image plane. SAM is expressed as an angular error which can be presented in degrees and smaller SAM values are preferable. An alternative measure, sometimes preferred in the remote sensing community, is the relative dimensionless global error in synthesis (ERGAS) defined as

$$
\operatorname{ERGAS}(\grave{\mathbf{f}}, \hat{\mathbf{f}}) \triangleq 100 \frac{1}{q^{K}} \sqrt{\frac{1}{K} \sum_{i=1}^{K}\left(\frac{\operatorname{RMSE}\left(\grave{f}_{i}, f_{i}\right)}{\mu\left(f_{i}\right)}\right)^{2}},
$$

where $\operatorname{RMSE}($,$) is the root mean squared error, K$ is the number of image sub-bands, and $\mu(\cdot)$ is the mean value of an image sub-band. Finally, the universal image quality index $Q$ defined in [28] and its extension [29], $Q 4$, that aims to estimate jointly the quality of four band imagery using a quaternion representation, are also utilized for the benchmarking. Both the $Q$ and $Q 4$ are averaged over sliding window of size $W \times W$, thus we indicate the window size by a subscript as $Q_{W}$ or $Q_{W}^{4}$.

Table 1 compares the quantitative performance of the different algorithms using the different quality measures, where the best score for each measure is shown in bold. From the numerical measures we see that the proposed technique does quite well. Results for other image datasets in the GeoEye dataset [24] exhibit the same trend, the proposed method consistently performs better than the alternative methods benchmarked here with respect to each of the measures, with the exception of the SNR for the red channel, where it does a little worse and the blue channel SNR, where it is comparable to the best but not always the best. Because several of the measures vary significantly over the different images (in particular SNR and SAM), we do not present average performance over the images. The better performance of the proposed technique with respect to the joint measures ERGAS and Q4 highlights a benefit of the joint formulation: while for individual channel pan-sharpening techniques, the pan-sharpening of one channel does not exercise an influence on the other, for the proposed method the joint formulation better imposes the requirement of spectral consistency. Visual comparison further emphasizes the advantage of the proposed technique over the other alternatives, a small corresponding region of the ground truth and the images obtained by three alternatives, is shown in Fig. 2 (for R, $\mathrm{G}, \mathrm{B}$ bands combined as a composite image). The images are best viewed in the electronic version of the paper (hopefully unaltered by post processing in the review process), at a high zoom.

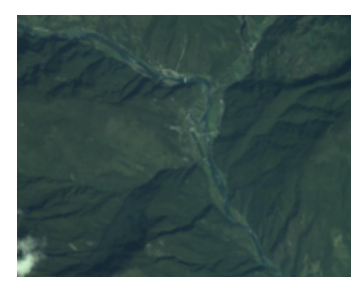

(a) Bicubic Interpolation

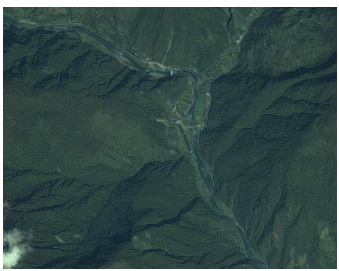

(c) Proposed Method

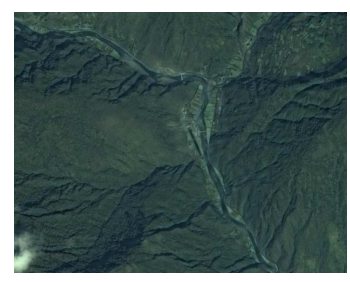

(b) Multi (4) level Wavelets [6]

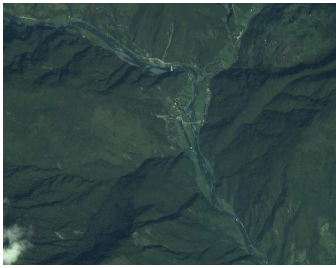

(d) Ground Truth
Fig. 2. Three (R, G, B) band presentation of a small cropped region of the ground truth and images obtained by three of the algorithms.

\section{CONCLUSION}

A novel joint formulation is introduced for pan-sharpening as the joint determination of the high resolution spectral images to minimize a joint objective function that combines squared residual error in physically motivated observation models of the low resolution multispectral and the high resolution panchromatic images. A computationally tractable iterative algorithm is introduced for solving the resulting optimization. Benchmark results ${ }^{8}$ demonstrate that the algorithm offers an improvement over commonly employed prior methods.

\footnotetext{
${ }^{8}$ We thank the Center for Integrated Research Computing, University of Rochester, for making available computational resources required for obtaining the results presented in this paper.
} 


\section{REFERENCES}

[1] T. Carper, T. Lillesand, and R. Kiefer, "The use of intensity-huesaturation transformations for merging SPOT panchromatic and multispectral image data," Photogram. Eng. Remote Sens., vol. 56, p. 459, 1990.

[2] K. Edwards and P. Davis, "The use of intensity-hue-saturation transformation for producing color shaded-relief images," Photogram. Eng. Remote Sens., vol. 60, p. 1369, 1994.

[3] T. Tu, S. Su, H. Shyu, and P. Huang, "A new look at IHS-like image fusion methods," Elsevier: Inf. fusion, vol. 2, pp. 177-186, 2001.

[4] C. Laben and B. Brower, "Process for enhancing the spatial resolution of multispectral imagery using pan-sharpening," U.S. Patent 6011875, Eastman Kodak Company, Jan. 2000.

[5] J. Zhou, D. Civco, and J. A. Silander, "A wavelet transform method to merge Landsat TM and SPOT panchromatic data," Intern. J. Remote Sens., vol. 19, pp. 743-757, Apr. 1998.

[6] T. Ranchin and L. Wald, "Fusion of high spatial and spectral resolution images: The ARSIS concept and its implementation," Photogram. Eng. Remote Sens., vol. 66, pp. 4-18, 2000.

[7] K. Vani and P. Ramya, "Fusion of satellite images using multiwavelet transform," in Proc. IEEE Int. Conf. Signal Processing Communications and Networking, Jan. 2008, pp. 306-310.

[8] M. Choi, R. Kim, M. Nam, and H. Kim, "Fusion of multispectral and panchromatic satellite images using the curvelet transform," IEEE Geosci. Remote Sens. Lett., vol. 2, pp. 136-140, Apr. 2005.

[9] H. Aanæs, J. Sveinsson, A. Nielsen, T. Bøvith, and J. Benediktsson, "Model-based satellite image fusion," IEEE Trans. Geosci. Remote Sens., vol. 46, no. 5, pp. 1336-1346, May 2008.

[10] Z. Li and H. Leung, "Fusion of multispectral and panchromatic images using a restoration-based method," IEEE Trans. Geosci. Remote Sens., vol. 47, no. 5, pp. $1482-1491$, may 2009.

[11] T. Wan, N. Canagarajah, and A. Achim, "Compressive image fusion," in Proc. IEEE Int. Conf. Image Processing, Oct. 2008, pp. 1308-1311.

[12] D. Liu and P. Boufounos, "Dictionary learning based pan-sharpening," in Proc. IEEE Intl. Conf. Acoustics Speech and Sig. Proc. Kyoto, Japan: IEEE, Mar. 2012, pp. 2397-2400.

[13] J. G. Liu, "Smoothing filter-based intensity modulation: a spectral preserve image fusion technique for improving spatial details," Intern. J. Remote Sens., vol. 21, pp. 3461-3472, Apr. 2000.

[14] Y. Zhang, "Understanding image fusion," Photogram. Eng. Remote Sens., vol. 70, p. 657, 2004.

[15] Z. Wang, D. Ziou, C. Armenakis, D. Li, and Q. Li, "A comparative analysis of image fusion methods," IEEE Trans. Geosci. Remote Sens., vol. 43, no. 6 , pp. 1391-1402, June 2005.

[16] C. Thomas, T. Ranchin, L. Wald, and J. Chanussot, "Synthesis of multispectral images to high spatial resolution: a critical review of fusion methods based on remote sensing physics," IEEE Trans. Geosci. Remote Sens., vol. 46, no. 5, pp. 1301-1312, May 2008.

[17] L. Alparone, L. Wald, J. Chanussot, C. Thomas, P. Gamba, and L. Bruce, "Comparison of pansharpening algorithms: outcome of the 2006 GS-S data-fusion contest," IEEE Trans. Geosci. Remote Sens., vol. 46, no. 5, pp. 1301-1312, May 2008.

[18] K. Nikolakopoulos, "Comparison of four different fusion techniques for IKONOS data," in Proc. IEEE Int. Symp. Geoscience and Remote Sensing, vol. 4, Sept. 2004, pp. 2534-2537.

[19] S. Park, M. Park, and M. Kang, "Super-resolution image reconstruction: a technical overview," IEEE Sig. Proc. Mag., vol. 20, no. 3, pp. 21-36, Mar. 2003.

[20] R. C. Gonzalez and P. Wintz, Digital Image Processing, 2nd ed. Reading, MA: Addison-Wesley, 1987.

[21] E. Dubois, "The sampling and reconstruction of time-varying imagery with application in video systems," Proc. IEEE, vol. 73, no. 4, pp. 502522, Apr. 1985.
[22] H. A. Aly and E. Dubois, "Specification of the observation model for regularized image up-sampling," IEEE Trans. Image Process., vol. 14, pp. 567-576, May 2005.

[23] D. G. Luenberger, Linear and Nonlinear Programming, 2nd ed. Reading, MA: Addison Wesley, 1989.

[24] S. Imaging, "Geoeye IKONOS scene data," 2000. [Online]. Available: ftp://ftp.glcf.umiacs.umd.edu/glcf

[25] M. Cook, B. Peterson, G. Dial, L. Gibson, F. Gerlach, K. Hutchins, R. Kudola, and H. Bowen, "IKONOS technical performance assessment," in Aerospace/Defense Sensing, Simulation, and Controls. International Society for Optics and Photonics, 2001, pp. 94-108. [Online]. Available: http://www.spaceimaging.com

[26] A. Garzelli, F. Nencini, L. Alparone, B. Aiazzi, and S. Baronti, "Pansharpening of multispectral images: a critical review and comparison," in Proc. IEEE Int. Symp. Geoscience and Remote Sensing, vol. 1, sept. 2004, pp. -84 .

[27] B. Aiazzi, S. Baronti, and M. Selva, "Improving component substitution pansharpening through multivariate regression of MS+ Pan data," IEEE Trans. Geosci. Remote Sens., vol. 45, no. 10, pp. 3230-3239, 2007.

[28] Z. Wang and A. Bovik, "A universal image quality index," IEEE Signal Process. Lett., vol. 9, pp. 81-84, Mar. 2002.

[29] L. Alparone, S. Baronti, A. Garzelli, and F. Nencini, "A global quality measurement of pan-sharpened multispectral imagery," IEEE Geosci. Remote Sens. Lett., vol. 1, pp. 313-317, Oct. 2004. 